14.2

\title{
Фазовые переходы в самоорганизованных микротрубках дифенилаланина, индуцированные негидростатическим давлением
}

\author{
(c) A. Крылов, ${ }^{1}$ C. Крылова, ${ }^{1}$ S. Kоруl, ${ }^{2}$ A. Kholkin ${ }^{2}$ \\ ${ }^{1}$ Институт фризики им. Л.В. Киренского ФИЦ КНЦ СО РАН, \\ 660036 Красноярск, Россия \\ ${ }^{2}$ Department of Physics \& CICECO - Materials Institute of Aveiro, University of Aveiro, \\ 3810-193 Aveiro, Portugal \\ e-mail: slanky@iph.krasn.ru
}

(Поступило в Редакцию 18 декабря 2017 г.)

Исследованы структурные фазовые переходы в микротрубках дифенилаланина, вызванные увеличением негидростатического давления. Выполнены исследования методом комбинационного рассеяния света, интерпретация и анализ полученных данных. Проанализированы спектральные изменения в области колебаний фенильных колец и высокочастотных колебаний NH- и СН-групп. Показано, что в области давлений до $9.8 \mathrm{GPa}$ наблюдаются четыре спектральные аномалии, указывающие на наличие фазовых переходов. Переходы при давлениях 1.7 и $4 \mathrm{GPa}$ обратимы. Переход при $5.7 \mathrm{GPa}$ происходит с частичной аморфизацией образца.

DOI: $10.21883 /$ JTF.2018.09.46420.2606

Пептиды привлекают внимание исследователей благодаря их биосовместимости, а также уникальным биологическим и физическим свойствам. Самая маленькая пептидная последовательность это дифенилаланин. Дифенилаланин самоорганизуется, формируя производные дипептидные наноструктуры, включая трубки, сферы, пластины и гидрогели [1]. Такие структуры обладают очень хорошей температурной и химической стабильностью [2]. Пептидные нанотрубки обладают уникальными свойствами, которые позволяют использовать их в качестве основного элемента наноэлектроники. Например, к таким свойствам относятся пьезоэффект, пироэффект [1-4]. Этот материал уже используется для создания пьезорезонаторов, микросоленоидов, батарей $[1,5-7]$. Внимание исследователей привлекают также материалы с новыми интересными свойствами, которые созданы при помощи сжатия пептидных микротрубок [8]. Поэтому целью настоящей работы стало исследование возможных структурных фазовых переходов микротрубок дифенилаланина при повышении давления. Надо отметить, что спектроскопия комбинационного рассеяния (КР) света широко используется для исследований структурных фазовых переходов [9-13], поэтому она применялась в настоящей работе в качестве методики исследования.

Для получения спектров КР в качестве источника возбуждения было использовано поляризованное излучение $\mathrm{Ar}^{+}$-лазера с длиной волны $514.5 \mathrm{~nm}$. Спектры в геометрии $180^{\circ}$ были получены на спектрометре Horiba Jobin Yvon T64000. Эксперименты при повышении давления были выполнены при температуре $295 \mathrm{~K}$ в ячейке высокого давления (Diacell $\mu \operatorname{ScopeDAC-HT}(\mathrm{G})$ ). Давление определялось по сдвигу полосы люминесценции ${ }^{5} D_{0}-{ }^{7} F_{0}$ иона самария $\mathrm{Sm}^{2+}$ в кристалле $\mathrm{SrB}_{4} \mathrm{O}_{7}: \mathrm{Sm}^{2+}$ [14-16]. Погрешность определения давления около $0.05 \mathrm{GPa}$.
Органический кристалл принадлежит пространственной группе $\mathrm{P6}_{1}$ [17]. Ячейка включает шесть молекул $\mathrm{NH}_{2}$ PhePheCOOH с 43 ионами в каждой молекуле. Ионы локализованы в позиции Уайкоффа 6а. Правила отбора в центре зоны Бриллюэна для комбинационного рассеяния света: $\quad \Gamma_{\text {Raman }}=128 A+129^{1} E_{2}+129^{2} E_{2}+$ $+128^{1} E_{1}+128^{2} E_{1}$, для инфракрасного поглощения: $\Gamma_{\mathrm{IR}}=128 A+128^{1} E_{1}+128^{2} E_{1}$, акустические моды: $\Gamma_{\text {Ас }}=A+{ }^{1} E_{1}+{ }^{2} E_{1}$, где ${ }^{1} E_{2},{ }^{2} E_{2}$ и ${ }^{1} E_{1},{ }^{2} E_{1}$ являются комплексно сопряженными представлениями продольных и поперечных мод. Внутри наноканалов присутствуют также молекулы воды [17].

На рис. 1 представлен полный спектр комбинационного рассеяния света при температуре $T=295 \mathrm{~K}$. Спектр можно разделить на три области, соответствующие колебаниям структурных элементов: а) $10-220 \mathrm{~cm}^{-1}$ решеточные колебания; b) $220-1800 \mathrm{~cm}^{-1}$ - внутренние колебания групп, включающие колебания $\mathrm{COO}$, $\mathrm{C}-\mathrm{C}$-групп, линия $1002 \mathrm{~cm}^{-1}$, соответствующая дыхательной моде фенильных колец, линии в области $1032 \mathrm{~cm}^{-1}$, соответствующие деформационным колебаниям $\mathrm{C}-\mathrm{H}$, колебания групп $\mathrm{NH}_{3}$; с) $2800-3250 \mathrm{~cm}^{-1}-$ валентные колебания групп С-H и $\mathrm{N}-\mathrm{H}$ [9].

Линия $1002 \mathrm{~cm}^{-1}$ соответствует дыхательным колебаниям ароматического кольца. Это самая интенсивная линия в спектре. Изменения в этой области спектра показаны на рис. 2, $a-c$ повышением давления, $b-c$ понижением давления. При понижении давления спектры не повторяются полностью, что указывает на необратимость структурных изменений. Чтобы проследить изменения в спектре, найдены зависимости смещения линий (рис. 3,a) и ширин линий (рис. 3,b) от давления. Черными треугольниками обозначены позиция (рис. 3,a) и ширина (рис. $3, b$ ) линии $1036 \mathrm{~cm}^{-1}$, черными кругами обозначены позиция (рис. 3,a) и ширина (рис. $3, b)$ линии $1032 \mathrm{~cm}^{-1}$, белыми квадратами - 

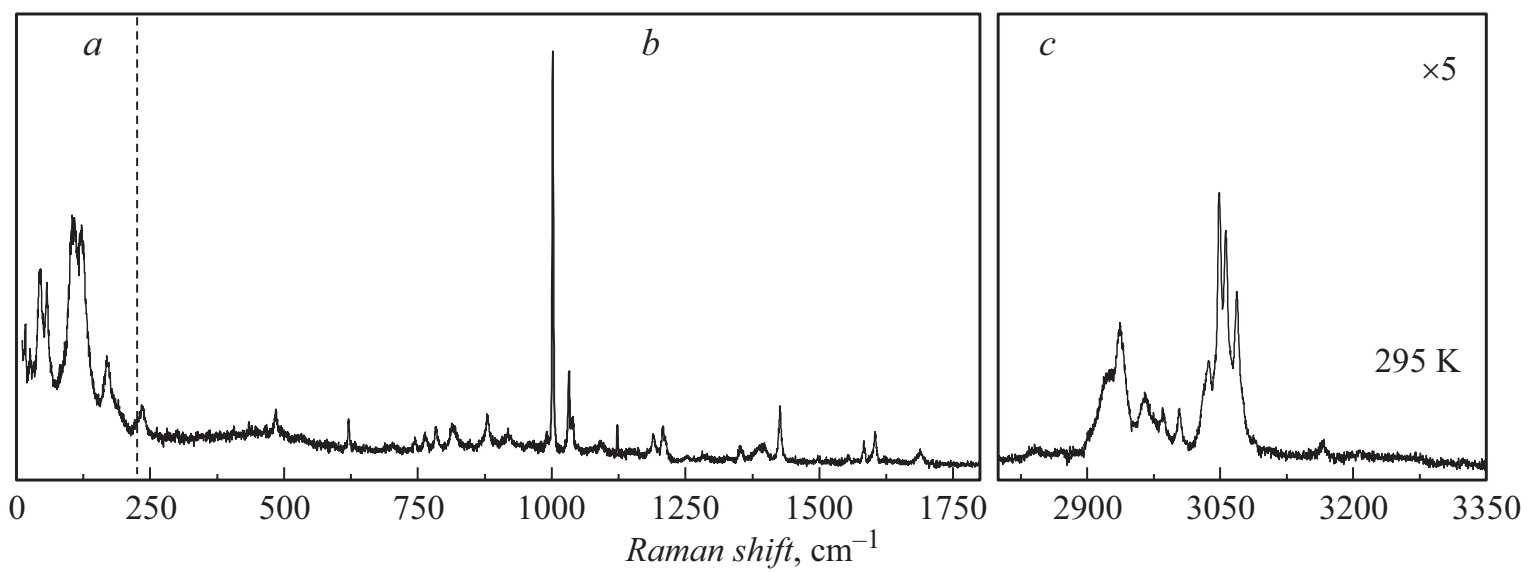

Рис. 1. Полный спектр комбинационного рассеяния света при температуре $T=295 \mathrm{~K}$ и нормальном атмосферном давлении $(P=0 \mathrm{GPa})$.
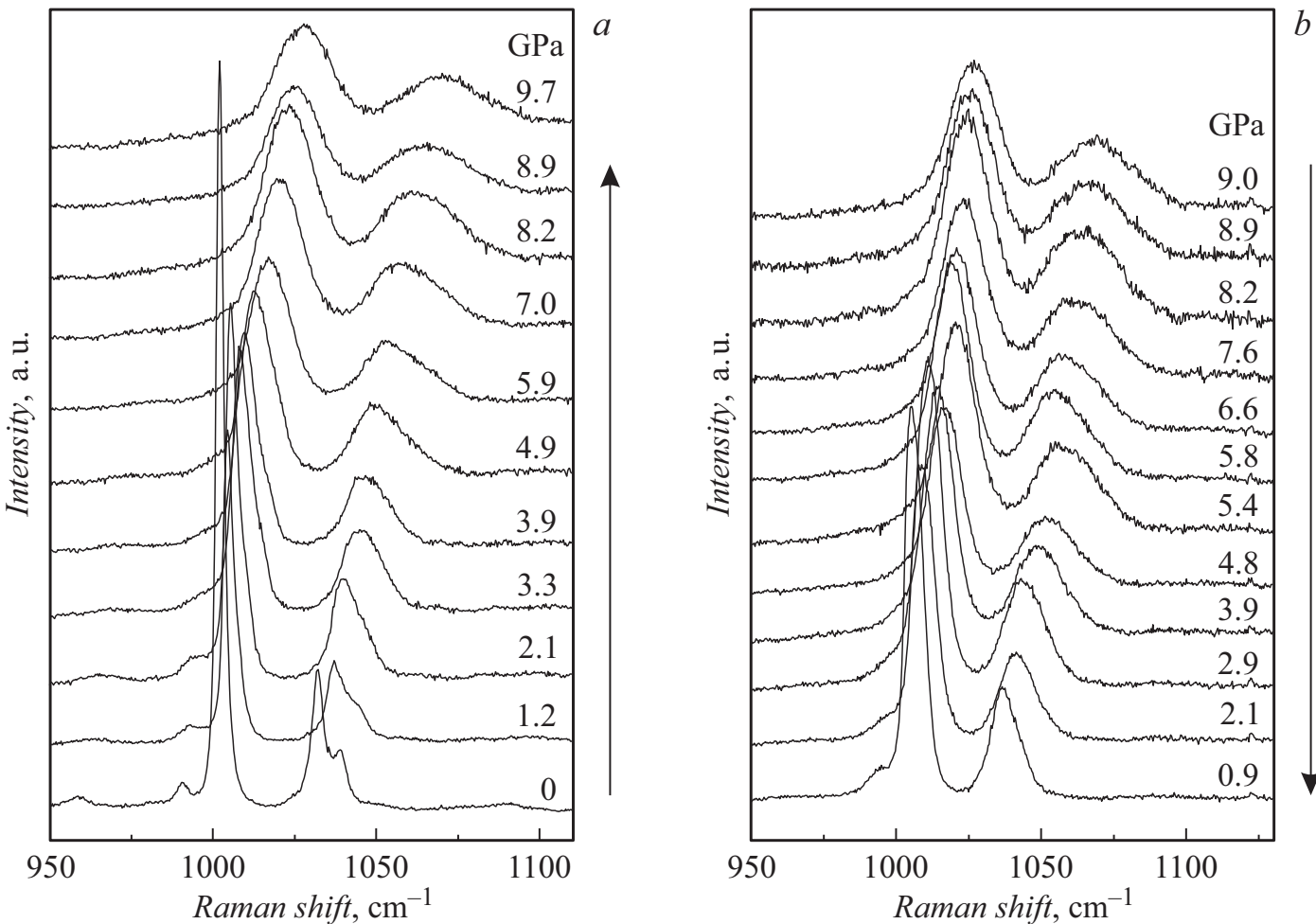

Рис. 2. Трансформация спектра в области колебаний фенильного кольца: $a-$ при повышении давления, $b-$ при снижении давления.

позиция (рис. $3, a$ ) и ширина (рис. $3, b$ ) линии $1002 \mathrm{~cm}^{-1}$. Черными треугольниками с вершинами, направленными вниз, обозначены позиция (рис. $3, a$ ) и ширина (рис. $3, b$ ) линии $1003 \mathrm{~cm}^{-1}$, обнаруженной при повышении давления до $1.1 \mathrm{GPa}$. По поведению этих параметров можно предположить, что структурные изменения происходят при давлениях 2, 5.7 GPa и, возможно, $4 \mathrm{GPa}$. Для уточнения возможного третьего перехода было построено отношение интенсивностей линий 1002 и $1032 \mathrm{~cm}^{-1}$. На рис. 3, с показан результат, подтверждающий наличие фазовых переходов при давлениях 1.7, 4, 5.7 GPa.
Полосы 1032 и $1038 \mathrm{~cm}^{-1}$ соответствуют продольным колебаниям фенильного кольца в плоскости. Молекула содержит два практически идентичных ароматических кольца, эти два колебания расщепляются в спектре в присутствии воды. Расстояние между колебаниями позволяет оценить количество молекул воды внутри кольца из 6 молекул дифенилаланина [18]. Поведение расстояния между этими двумя пиками показано на рис. 4. Мы наблюдаем, что расстояние между пиками уменьшается вплоть до $1.7 \mathrm{GPa}$, затем оно начинает расти до $4 \mathrm{GPa}$, далее продолжает расти, но изменя- 

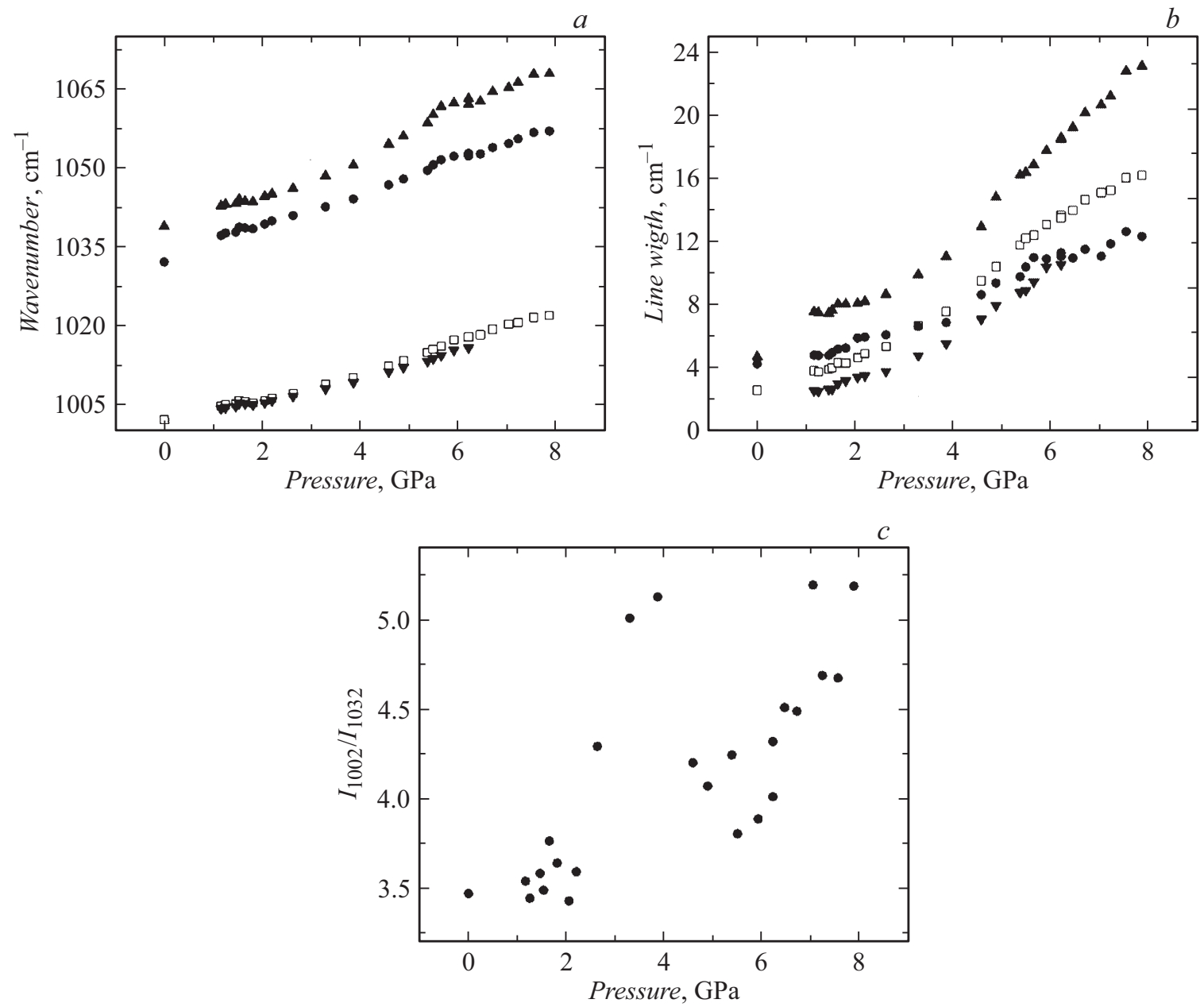

Рис. 3. Зависимость $a-$ смещения позиций линий, $b-$ ширины линий и $c$ - отношения интенсивностей от температуры в области, соответствующей колебаниям фенильного кольца.

ется угол наклона кривой зависимости. При $5.7 \mathrm{GPa}$ изменения угла наклона также указывают на фазовый переход.

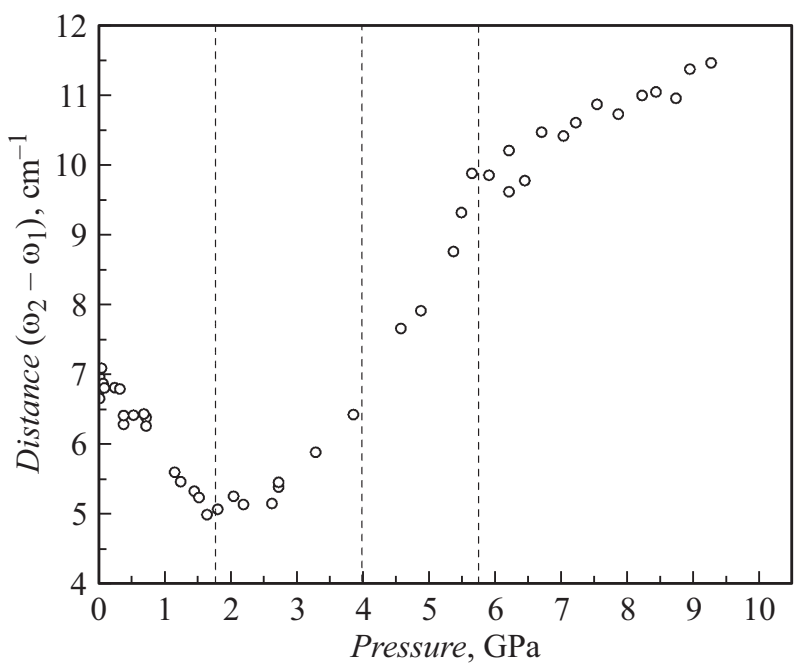

Рис. 4. Расстояние между пиками $\sqcup_{1}=1032$ и $\sqcup_{2}=1038 \mathrm{~cm}^{-1}$ в зависимости от давления.
В высокочастотной области спектра, связанной с колебаниями групп $\mathrm{C}-\mathrm{H}$ и $\mathrm{N}-\mathrm{H}$ также проявляются изменения, связанные с фазовыми переходами. На рис. 5, $a$ показана трансформация спектров с увеличением давления. При нормальных условиях (без приложения давления) наблюдается 7 линий. В спектре при увеличении давления остаются две широкие линии. На рис. 5, $b$ построена зависимость позиций линий от давления. Штриховыми линиями показаны спектроскопические изменения, указывающие на наличие фазовых переходов: изменение количества линий и угла наклона смещения позиций линий. Изменения в спектрах указывают на существование четвертого фазового перехода при давлении $P_{4}=7 \mathrm{GPa}$ (рис. $5, b$ ).

В результате экспериментальных наблюдений были найдены четыре критические точки при давлениях $P_{1}=1.7, P_{2}=4, P_{3}=5.7, P_{4}=7 \mathrm{GPa}$. До первой критической точки происходило сжатие трубок без разрушения кристаллической структуры. Первый и второй фазовый переход являются обратимыми. Тем не менее даже повышение давления выше $6 \mathrm{GPa}$ не привело к полной аморфизации. Количество наблюдаемых фазовых 

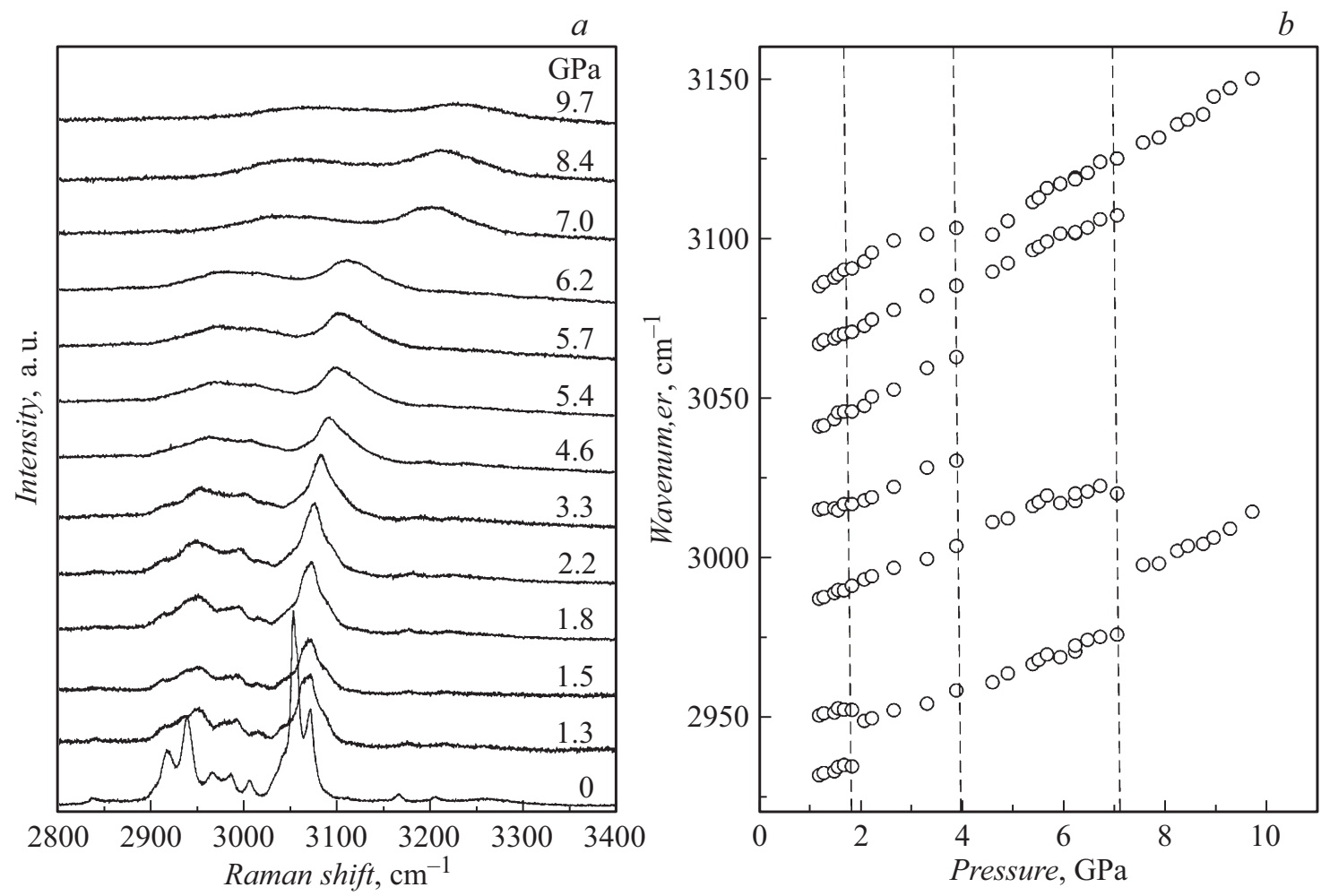

Рис. 5. Высокочастотные колебания: $a$ - трансформация спектров при повышении давления, $b-$ зависимость смещения позиций линий от давления.

переходов в нашем исследовании больше, чем в работе [10]. Это связано с тем, что нами исследован более широкий диапазон давлений при негидростатическом сжатии. Кроме того, в нашей работе в рассмотрение включены высокочастотные области спектров, связанные с колебаниями групп $\mathrm{C}-\mathrm{H}$ и $\mathrm{N}-\mathrm{H}$, которые не рассматривались подробно в работе [10]. Полученные нами данные полезны как для создания новых материалов, так и в качестве понимания основных физических свойств микротрубок. Однако следует провести дальнейшее исследование по определению структур фаз высокого давления пептидных трубок методами рентгеноструктурного анализа.

Авторы благодарят Turkish-Portuguese Project No.TUBITAK/0006/2014 за финансовую поддержку.

\section{Список литературы}

[1] Adler-Abramovicha L., Ehud Gazit E. // Chem. Soc. Rev. 2014. Vol. 43. P. 6881.

[2] Adler-Abramovich L., Aronov D., Beker P., Yevnin M., Stempler S., Buzhansky L., Rosenman G., Gazit E. // Nat. Nanotechnol. 2009. Vol. 4. P. 849.

[3] Kholkin A., Amdursky N., Bdikin I., Gazit E., Rosenman G. // ACS NANO. 2010. Vol. 4. N 2. P. 610.

[4] Esin A., Baturin I., Nikitin A., Vasilev T., Salehli F., Shur V., Kholkin A. // Appl. Phys. Lett. Vol. 109. N 14. P. 142902.

[5] Bosne E.D., Heredia A., Kopyl S., Karpinsky D.V., Pinto A.G., Kholkin A.L. // Appl. Phys. Lett. 2013. Vol. 102. P. 073504.
[6] Nuraeva A., Vasilev S., Vasileva D., Zelenovskiy P., Chezganov D., Esin A., Kopyl S., Romanyuk K., Shur V., Kholkin A. // Crystal Growth \& Design. 2016. Vol. 16. N 3. P. 1472.

[7] Ryan K., Beirne J., Redmond G., Kilpatrick J.I., Guyonnet J., Buchete N.-V., Kholkin A.L., Rodriguez B.J. // ACS Appl. Mater. Interfaces. 2015. Vol. 7. N 23. P. 12702.

[9] Zelenovskiy P.S., Davydov A.O., Krylov A.S., Kholkin A.L. // JRS. 2017. Vol. 48. P. 1401.

[10] Filho J.G. da Silva, Filho J. Mendes Melo F.E.A., Lima J.A.Jr., Freire P.T.C. // Vibrational Spectroscopy. 2017. Vol. 92. P. 173.

[11] Krylov A.S., Goryainov S.V., Laptash N.M., Vtyurin A.N., Melnikova S.V., Krylova S.N. // Crystal Growth \& Design. 2014. Vol. 14. N 1. P. 374.

[12] Vtyurin A.N., Krylov A.S., Goryainov S.V., Krylova S.N., Oreshonkov A.S., Voronov V.N. // Phys. Sol. State. 2012. Vol. 54. N 5. P. 934.

[13] Vtyurin A.N., Goryalnov S.V., Zamkova N.G., Zinenko V.I., Krylov A.S., Krylova S.N. // Com. Mat. Sc. 2006. Vol. 36. N 12. P. 79

[14] Bdikin I., Bystrov V., Kopyl S., Lopes Rui P.G., Delgadillo I., Gracio J., Mishina E., Sigov A., Kholkin A. // Appl. Phys. Lett. 2012. Vol. 100. P. 043702.

[15] Krylov A., Gudim I., Nemtsev I., Krylova S.N., Shabanov A.V., Krylov A.A. // JRS. 2017. Vol. 48. P. 1406.

[16] Pugachev A., Zaytseva I., Krylov A., Malinovsky V., Surovtsev N., Borzdov Yu., Kovalevsky V. // Ferroelectrics. 2017. Vol. 508. N 1. P. 161.

[17] Gorbitz C.H. // Chem. Comm. 2006. N 22. P. 2332.

[18] Wu X., Xiong S., Wang M., Shen J., Chu P.K.J. // Phys. Chem. C. 2012. Vol. 116. P. 9793. 